\title{
The compression of L5 nerve root, single or double sites?- radiographic graded signs, intra-operative detect technique and clinical outcomes
}

\author{
Ai-Min Wu ${ }^{1,2 \#}$, Kai Zhang ${ }^{1 \#}$, Xun-Lin Li ${ }^{1 \#}$, Xiao-Fei Cheng ${ }^{1}$, Tang-Jun Zhou ${ }^{1}$, Lin Du ${ }^{1}$, Chen Chen ${ }^{1}$, Hai- \\ Jun Tian ${ }^{1}$, Xiao-Jiang Sun ${ }^{1}$, Chang-Qing Zhao ${ }^{1}$, Yan Michael Li $^{3}$, Jie Zhao ${ }^{1}$ \\ ${ }^{1}$ Department of Orthopaedic Surgery, Shanghai Ninth People's Hospital, Shanghai Jiao Tong University School of Medicine, Shanghai Key \\ Laboratory of Orthopaedic Implants, Shanghai 200011, China; ${ }^{2}$ Department of Spine Surgery, Zhejiang Spine Surgery Centre, Orthopaedic \\ Hospital, The Second Affiliated Hospital and Yuying Children's Hospital of the Wenzhou Medical University, The Second School of Medicine \\ Wenzhou Medical University, The Key Orthopaedic Laboratory of Zhejiang Province, Wenzhou 325035, China; ${ }^{3}$ Department of Neurosurgery, \\ University of Rochester School of Medicine and Dentistry, Rochester, NY, USA \\ \#These authors contributed equally to this work and are co-first authors on this work.
}

Correspondence to: Jie Zhao, MD, PhD. Department of Orthopaedic Surgery, Shanghai Ninth People's Hospital, Shanghai Jiao Tong University School of Medicine, Shanghai Key Laboratory of Orthopaedic Implants, 639 Zhizaoju Road, Shanghai 200011, China. Email: profzhaojie@126.com.

Background: The L5 nerve root could be compressed at both L4-5 and L5-S1 regions. If L5 nerve root has confirmed compression at L4-5 level and questionable compression at L5-S1 foramina, performing both surgeries at L4-5 and L5-S1 levels may induce unnecessary extra surgery on L5-S1; however, ignoring foraminal stenosis of L5/S1 may require re-exploration.

Methods: Two hundred seventeen patients with L5 nerve root compressed at L4-5 lateral access were performed with L4-5 decompression and interbody fusion. Lee et al. grade classification was used to assess the foraminal stenosis of L5-S1 preoperatively. Nerve root probe was designed and used to detect if there were foraminal stenosis at L5-S1 level that compressing the exiting L5 nerve root. Visual analog scale (VAS) of low back pain, leg pain and Oswestry Disability Index (ODI) were used to assess clinical outcomes.

Results: For all of 217 patients who underwent L4-5 surgery, L5-S1 foramina were preoperatively assessed as: grade 0: 125 cases, grade 1: 58 cases, grade 2: 23 cases, and grade 3: 11 cases. After intra-operative L5 nerve root detection, $11 / 11$ patients with grade 3 radiographic foraminal stenosis, $6 / 23$ (26.1\%) with grade 2 and 2/58 (3.4\%) who had grade 1 underwent L4-5 and L5-S1 transforaminal lumbar interbody fusion (TLIF), the others received only L4-5 TLIF. Compared to pre-operative baseline data, both L4-5 TLIF and L4-5 and L5-S1 TLIF groups had significant decreased VAS of low back pain and leg pain, and ODI at 3 and 24 months after operation.

Conclusions: We suggested that our novel nerve root probe combined with pre-operative radiographic grade may be helpful to surgeons to identify the single or double compression of L5 nerve root and make a more precise surgical strategy to improve surgical outcome than the method depended on pre-operative radiographic grade alone.

Keywords: Lumbar stenosis; foraminal stenosis; lumbar arthrodesis; decompression

Submitted Feb 20, 2018. Accepted for publication May 17, 2018.

doi: 10.21037/qims.2018.05.08

View this article at: http://dx.doi.org/10.21037/qims.2018.05.08 


\section{Introduction}

Lumbar stenosis is one of the most common spinal disorders, may induce clinical symptoms of pain in the buttock or lower extremity, and intermittent neurogenic claudication, with or without low back pain (1-3), the surgical intervention will be recommended if patients are with no response to conservative management (4-6).

The L4-5 and L5-S1 levels were common sites of lumbar stenosis; the L-5 nerve root could be compressed at L4-5 and/or L5-S1 regions. At L4-5 regions, the L5 nerve root could be compressed at lateral recess, while at L5-S1, the exiting L5 nerve root could be compressed by foraminal stenosis. In the majority of patients, the L5 nerve compression is at single site, but there are also many patients, who have L5 nerve compressed at both L4-5 lateral recess and $\mathrm{L} 5-\mathrm{S} 1$ foraminal stenosis, and it was reported that the lumbar foraminal stenosis was not rare (7-9). A cadaveric study found that the lumbar foraminal stenosis in 21 out of 100 lumbar foramina examined (10).

But the clinical L5/S1 foraminal stenosis was often unrecognized (9) and accounted as the main reason for the failed lumbar surgery with continued post-operative symptoms (11-13). The problem was that when we observed L5 nerve root was compressed at L4-5 lateral recess at pre-operative radiographic images, and we also found the same L5 nerve root was crushed at L5-S1 foramina. To perform both surgery on L4-5 and L5-S1 levels may induce unnecessary additional surgery on L5-S1. If we only performed the surgery at L4-5 level, some patients might still have continued post-operative symptoms at L5/S1, which might require secondary surgery.

To overcome this problem by taking a more precise surgery, we used an intra-operative nerve root probe and combined it with the pre-operative radiographic grade to detect whether there were L5-S1 foraminal stenosis and the need to extend to the L5-S1 surgery.

\section{Methods}

\section{Patient population}

Between January 2013 and October 2015, 217 patients (88 males and 129 females) were included in this study. All of the included patients had pre-operative anteroposterior and lateral films, CT scans, and MR images. L5 nerve root was compressed at L4-5 lateral access, and the symptoms were identified at L5 nerve root, needed to be performed L4-5 decompression and interbody fusion. However, parts of them were suspected of having foraminal stenosis of L5S1, which also compressed the L5 nerve root, and cannot be pre-operatively differentiated if the symptoms were caused by the compression at L4-5 lateral access (single site) or both L4-5 lateral access and foraminal stenosis of L5-S1 (double sites). The foraminal stenosis of L5-S1 was assessed according to the Lee et al. classification (14) (Figure 1):

(I) Grade 0: normal;

(II) Grade 1: mild degree of foraminal stenosis, perineural tissue obliteration surrounding nerve root in the transverse direction or vertical direction, without the morphologic change of the nerve root;

(III) Grade 2: moderate degree of foraminal stenosis, perineural tissue obliteration surrounding nerve root in both transverse and vertical direction, without the morphologic change of the nerve root;

(IV) Grade 3: severe degree of foraminal stenosis, nerve root collapse or morphologic change due to severe perineural tissue obliteration surrounding the nerve root.

All of the pre-operative MRIs were assessed by two independent senior spine surgeons. If they had any disagreement, the image will be discussed at the group research meeting.

\section{Surgical technique}

Nerve root probe used in present surgical procedure was shown as Figure 2. All surgeries performed by one senior surgeon. The transforaminal lumbar interbody fusion (TLIF) was performed at level L4-5, after the decompression of L4-5 was finished. The nerve root probe was used to identify whether the L5 nerve root was compressed at the L5-S1 intervertebral foramina (Figure 3). The nerve root probe was inserted into the L5-S1 intervertebral foramina alongside at the L 5 nerve root intraoperatively. If the nerve root probe could be inserted gently, can around the nerve root one round, we judged that the compression of L5 nerve root at the L5-S1 intervertebral foramina was not severe and there was no need to perform TLIF at L5/S1 immediately, only the L4-5 TLIF was performed (Figure 3). If the nerve root probe could not be inserted into the L5-S1 intervertebral foramina, combined with the pre-operative MR images and the symptoms of the patient, we judged that there had compression of L5 nerve at the L5-S1 intervertebral foramina. The L5-S1 TLIF with L5-S1 intervertebral foramina decompression would be performed as well as L4-5 TLIF (Figure 4). 


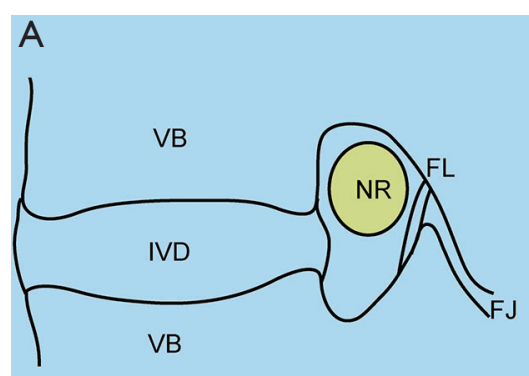

D

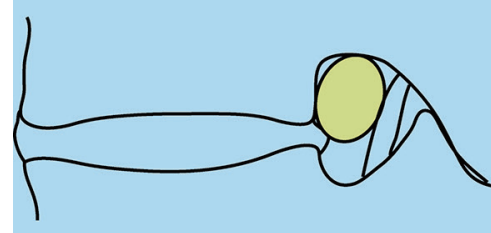

B

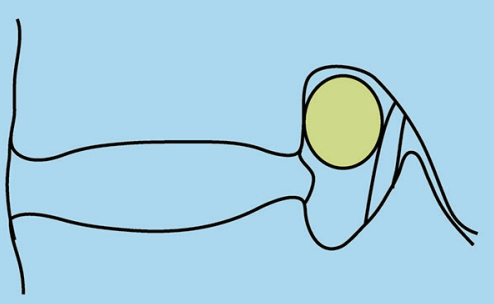

$\mathrm{E}$

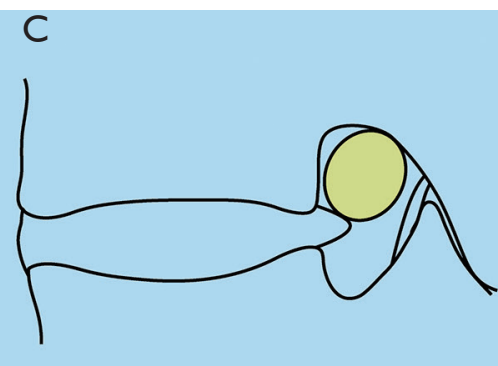

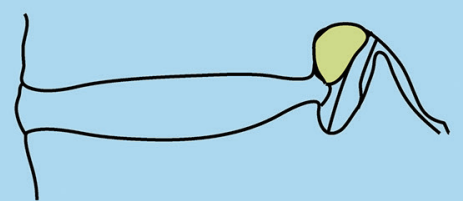

Figure 1 Schematic diagrams illustrating Lee et al. classification to assess the foraminal stenosis of L5-S1. (A) Grade 0: normal; (B) grade 1: mild degree of foraminal stenosis, perineural tissue obliteration surrounding nerve root in transverse direction, without the morphologic change of the nerve root; (C) grade 1: mild degree of foraminal stenosis, perineural tissue obliteration surrounding nerve root in vertical direction, without the morphologic change of the nerve root; (D) grade 2: moderate degree of foraminal stenosis, perineural tissue obliteration surrounding nerve root in both transverse and vertical direction, without the morphologic change of the nerve root; (E) grade 3 : severe degree of foraminal stenosis, nerve root collapse or morphologic change due to severe perineural tissue obliteration surrounding the nerve root. VB, vertebral body; IVD, intervertebral disc; NR, nerve root; FL, flavum ligamentum; FJ, facet joint.

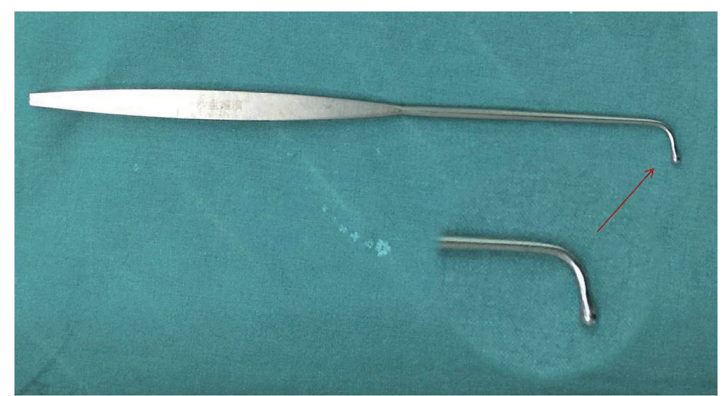

Figure 2 Photo of the nerve root probe. Arrow: the tip of the probe.

The duration of operative time, estimated blood loss, length of postoperative hospital stay were recorded and collected, as well as the intra-operative and post-operative complications such as dural tear, screw misplaced or failure, neurologic deficit, wound infection, hematoma, fusion failure and reoperation.

\section{Clinical outcomes}

Visual analog scale (VAS) of low back pain and leg pain was used to assess the pain outcomes; Oswestry Disability Index
(ODI) was used to assess the functional outcome. The VAS and ODI were assessed at time points of preoperatively, 3 -month after operation, and 24-month after operation by two independent researchers. Both of static and dynamic radiographs and CT scans were obtained at 3 and 24 months after surgery. Solid bony fusion was assessed on CT sagittal reconstruction images by continuity of the trabecular bony bridging across the disc space (15) and lateral dynamic flexion-extension radiographs that less than $3^{\circ}$ of angular motion between adjacent end plates of the indexed level $(16,17)$.

\section{Statistical analysis}

The data was analyzed by SPSS software (Version 17.0, SPSS Inc., Chicago, Illinois, USA). The data of preoperation, 3-month after operation, 24-month after operation was tested by the "repeated-measures analysis of variance" (ANOVA), the comparisons of duration of operative time, estimated blood loss, length of postoperative hospital stay, VAS of low back pain, VAS of leg pain and ODI were examined by independent samples $t$-test. The level of significance was set at $\mathrm{P}<0.05$. 

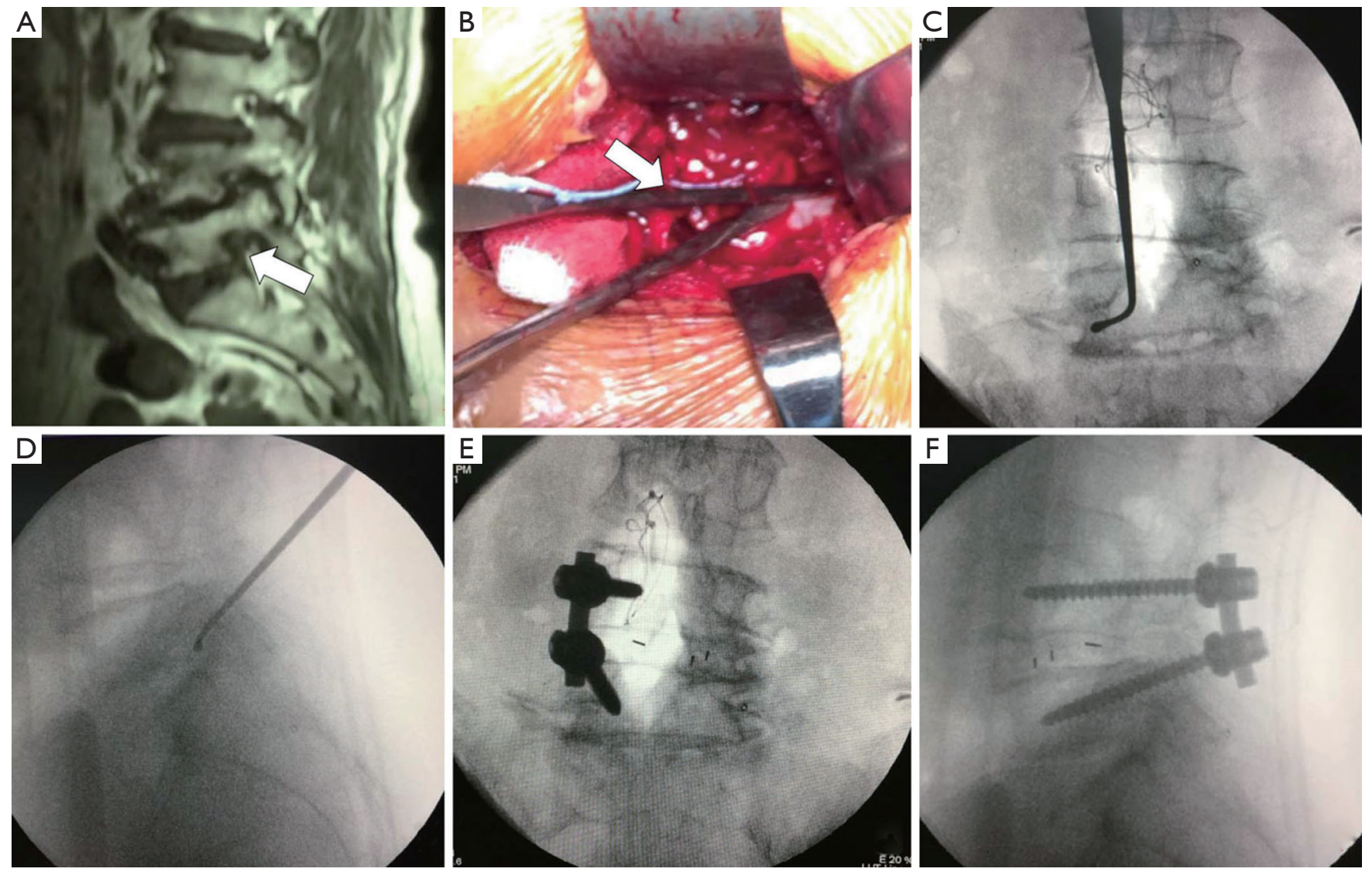

Figure 3 A patient with L4-5 lumbar stenosis undergoes L4-5 TLIF, and the pre-operative MRI (A) shows there is "grade 2" foraminal stenosis at L5-S1 level (arrow: the L5-S1 foramina). (B,C,D) After the decompression of L4-5, nerve root probe (arrow: the probe) is used to detect whether the L5 nerve root is compressed at the L5-S1 intervertebral foramina, and the detect tip can be inserted into the L5-S1 intervertebral foramina alongside at the L5 nerve root easily. Therefore, only L4-5 TLIF is performed (E,F). TLIF, transforaminal lumbar interbody fusion.

\section{Results}

A total of 217 patients were included in this study, with 88 males and 129 females, aged $63.8 \pm 10.8$ years old. In preoperative assessment of sagittal MR images, the distribution of Lee et al. classification (14) of L5-S1 foraminal stenosis is shown in Figure 5: grade 0: 125 cases, grade 1: 58 cases, grade 2: 23 cases, and grade 3: 11 cases.

After intra-operative L5 nerve root detection, we found that the detected tip cannot be inserted into the L5$\mathrm{S} 1$ foraminal stenosis in all 11 patients, who had grade 3 radiographic foraminal stenosis, $6 / 23(26.1 \%)$ who had grade 2 and $2 / 58$ (3.4\%) who had grade 1, we performed L4-5 and L5-S1 TLIF on these 19 patients. And the other 198 patients were only performed L4-5 TLIF. The detailed perioperative data was showed in Table 1.
Eight patients lost at follow up, and 209 patients (191 of L4-5 TLIF patients and 18 of L4-5 and L5-S1 TLIF patients) had at least 24 months follow up. No additional reoperation was performed on L4-5 and L5-S1 TLIF patients. For one patient who had grade 2 at pre-operation although, the detecting probe tip could be inserted into the L5-S1 foraminal stenosis at primary surgery, so he underwent only L4-5 TLIF; however the L5-S1 foraminal stenosis was progressed at 17 months after surgery, additional L5-S1 TLIF was performed. Three patients had fusion failure in L4-5 TLIF group and one in L4-5 and L5-S1 TLIF group; these four patients had no symptom so no additional operation was performed.

Compared to pre-operative baseline data, both L4-5 TLIF and L4-5 and L5-S1 TLIF groups had significant decreased VAS of low back pain and leg pain, and ODI at 

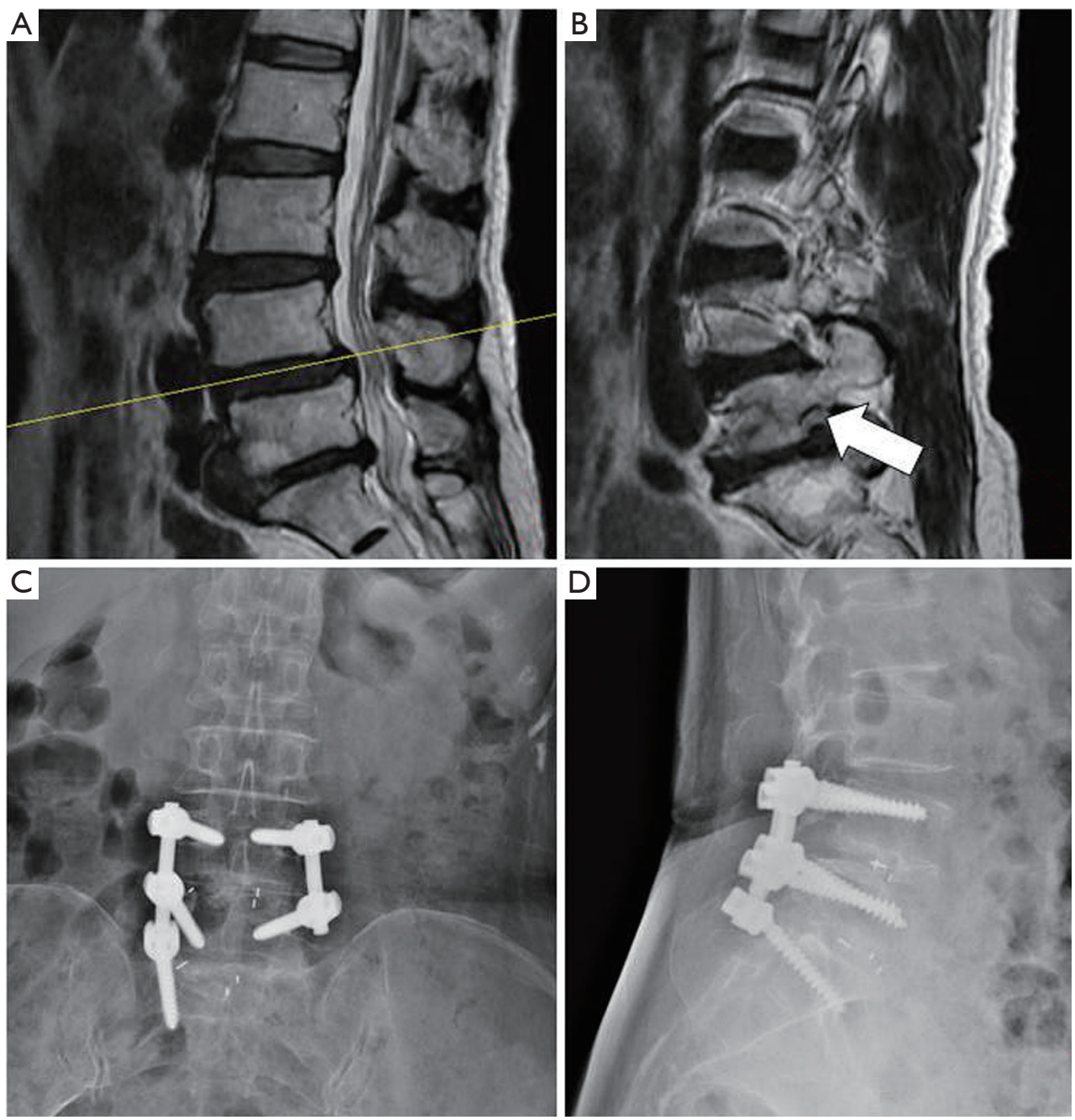

Figure 4 A patient with L4-5 degenerative lumbar spondylolisthesis with stenosis needs to perform the L4-5 TLIF (A) and the pre-operative MRI shows there is "grade 3" foraminal stenosis at L5-S1 level (B) (arrow: the L5-S1 foramina). Our novel designed nerve root probe cannot be inserted into the L5-S1 intervertebral foramina. Finally, only L4-5 TLIF with bilateral pedicle screw fixation and L5-S1 LTIF with unilateral pedicle screw fixation are performed (C: anteroposterior view; D: lateral view). TLIF, transforaminal lumbar interbody fusion.

3 months and 24 months after operation. The detailed VAS of low back pain, leg pain and ODI data were summarized in Table 2.

\section{Discussion}

The factors that compress the nerve root are variable, including the intervertebral disc herniation, spondylolisthesis, hypertrophy of ligamentum flavum and hypertrophy of facet joint etc. $(4,18-20)$. The most common sites of nerve root compressed are at levels of L4-5 and L5-S1. The compressing factors can be at lateral recess and/or foramina; they are at different adjacent lumbar levels for one nerve root. L5 nerve root is the most common one that could be compressed. In most case, the compression only occurs at the lateral recess of L4-5 level, and the foraminal stenosis of L5S1 often is ignored (9), and need to be re-explored $(21,22)$.

Lee et al. (14) developed a foraminal stenosis classification based on pre-operative MR images, which may help us to have a judgment before operation. However, the Lee classification doesn't provide the information that which grade stenosis need surgery, especially for patients whose L5 nerve root is compressed at L4-5 level and the L5-S1 foramina assessed as grade 1 and 2 (23), which will cause 


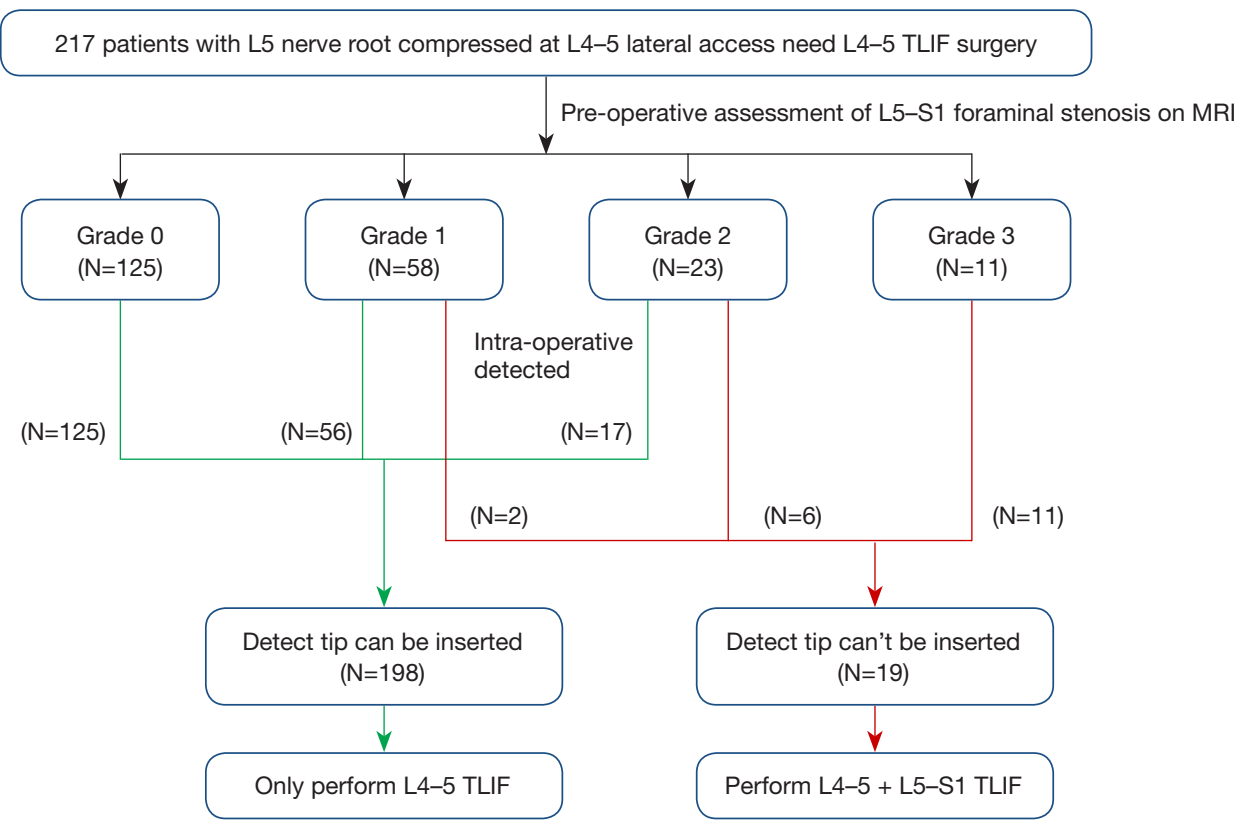

Figure 5 In preoperative MR assessment of L5-S1 foraminal stenosis and the final surgical perform on the included 217 patients.

Table 1 The perioperative data between two groups

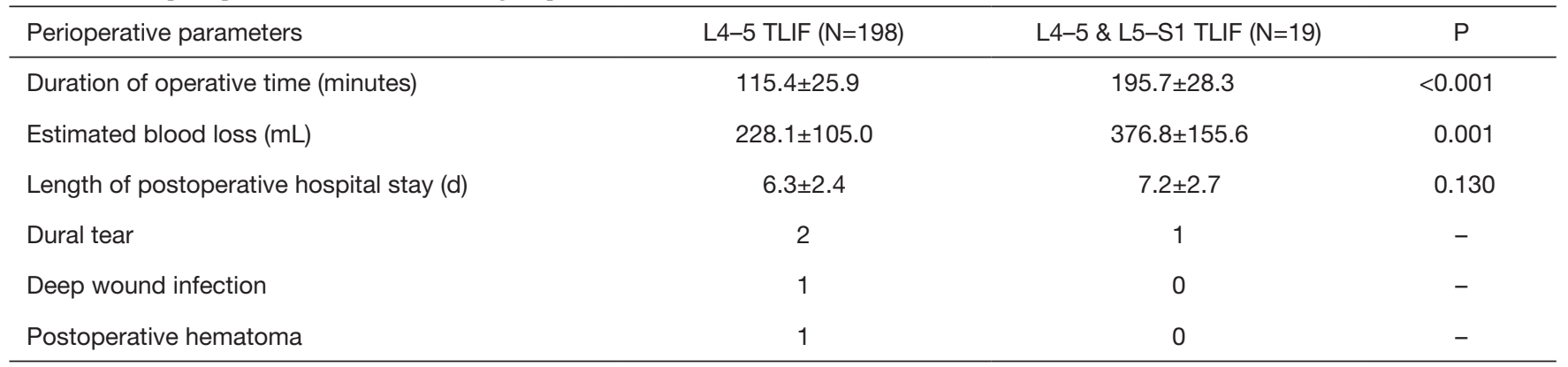

TLIF, transforaminal lumbar interbody fusion.

dilemma for surgeons of how to decide only decompression at L4-5 or extension to L5-S1. If the both L4-5 and L5-S1 TLIF are performed, it will be over treat for many patients. If only receiving the L4-5 level some patients may still have continued post-operative symptoms, and require secondary surgery. To make a more accurate decision, we combine the Lee classification (14) and intra-operative nerve root probe, which can help us to avoid the over treat and cutback the potential secondary surgery.

In our current study, among 58 patients assessed as grade 1 by pre-operative MRI, nerve root probe could not be inserted into foramina in 2 of them; nerve root probe could not be inserted into foramina in 6 of 23 patients assessed as grade 2. So both L4-5 and L5-S1 TLIF were performed on them. If the foramina assessed as grade 0 pre-operatively, the nerve root probe could be inserted into the foramina easily. We couldn't be inserted into foramina in patients assessed grade 3 pre-operatively. Therefore, we suggest the nerve root probe will be helpful for surgical strategy for patients that pre-operatively assessed as grade 1 or 2 on MRI.

The two-year follow-up found both the patients treated by only L4-5 TLIF and L4-5 and L5-S1 TLIF had significant improvement of VAS of low back pain, leg pain and ODI. We also found that total 73 patients, including 56 out of $58(96.6 \%)$ from patients assessed as grade 1 foraminal stenosis and 17/23 (73.9\%) patients assessed as grade 2, did not receive the additional L5-S1 surgery. And in these 73 patients, only one patient whose L5- 
Table 2 The data of clinical outcomes from baseline to two years follow-up

\begin{tabular}{lcc}
\hline Clinical parameters & $\begin{array}{c}\text { L4-5 TLIF } \\
(\mathrm{N}=191)\end{array}$ & $\begin{array}{c}\text { L4-5 \& L5-S1 } \\
\text { TLIF }(\mathrm{N}=18)\end{array}$ \\
\hline VAS of low back pain & & \\
Baseline & $6.3 \pm 1.8$ & $6.4 \pm 2.3$ \\
3 months after operation & $1.7 \pm 1.0^{*}$ & $1.9 \pm 1.2^{*}$ \\
24 months after operation & $1.5 \pm 1.0^{*}$ & $1.7 \pm 1.4^{*}$ \\
VAS of leg pain & & \\
Baseline & $6.9 \pm 1.4$ & $6.8 \pm 1.4$ \\
3 months after operation & $1.9 \pm 1.2^{*}$ & $1.8 \pm 1.1^{*}$ \\
24 months after operation & $1.7 \pm 1.0^{*}$ & $1.6 \pm 1.0^{*}$ \\
ODI & & \\
Baseline & $63.2 \pm 11.5$ & $61.1 \pm 8.5$ \\
3 months after operation & $31.1 \pm 5.9^{*}$ & $32.6 \pm 6.8^{*}$ \\
24 months after operation & $29.8 \pm 7.5^{*}$ & $29.3 \pm 6.0^{*}$ \\
\hline
\end{tabular}

*, $\mathrm{P}<0.05$ compare to baseline data. TLIF, transforaminal lumbar interbody fusion; VAS, visual analog scale; ODI, Oswestry Disability Index.

S1 foraminal stenosis was progressed at 17 months after surgery, and was treated by additional L5-S1 TLIF.

There were several limitations in our present study. Firstly, this was not a randomized controlled study or compared study, whether patients treated by only at level of L4-5 or extension to L5-S1 level were based on the intraoperative judge, because of the extend or not to L5-S1 fusion is varied for different surgeons, without a standard guideline, therefore, the number of patients benefited from our method could not be calculated accurately, only one patient needed re-exploration in our 217 patients for L5-S1 foraminal stenosis, which is lower than the literatures $(21,22)$; Secondly, the sample in the group of surgical extension to level of L5-S1 was small, because the extent surgery was based on strict intra-operative detection in order to avoid unnecessary additional surgery, most cases [17/23 (73.9\%) of grade 2 patients] were avoided the additional L5-S1 fusion and with good clinical outcomes. Thirdly, the 2-year followup was still the short-term result, in the future mid-term and long-term follow-up are needed to be conducted.

\section{Conclusions}

Our current study found that our novel nerve root probe combined with pre-operative radiographic graded may be helpful for us to differentiate the single or double compression of L-5 nerve root and make a more precise surgical strategy to improve surgical outcome than the method depended on pre-operative radiographic graded alone.

\section{Acknowledgements}

Funding: This work was supported by the National Natural Science Foundation of China (81501933, 81572168), Wenzhou leading talent innovative project (RX2016004), Zhejiang Provincial Medical Technology Foundation of China (2018KY129), China Postdoctoral Science Foundation (2018M630450).

\section{Footnote}

Conflicts of Interest: The authors have no conflicts of interest to declare.

Ethical Statement: This study was approved by Institutional Review Board (IRB) (No. 2015-37) of Ninth People's Hospital Shanghai Jiao Tong University School of Medicine.

\section{References}

1. Watters WC 3rd, Baisden J, Gilbert TJ, Kreiner S, Resnick DK, Bono CM, Ghiselli G, Heggeness MH, Mazanec DJ, O'Neill C, Reitman CA, Shaffer WO, Summers JT, Toton JF; North American Spine Society. Degenerative lumbar spinal stenosis: an evidence-based clinical guideline for the diagnosis and treatment of degenerative lumbar spinal stenosis. Spine J 2008;8:305-10.

2. Lurie J, Tomkins-Lane C. Management of lumbar spinal stenosis. BMJ 2016;352:h6234.

3. Katz JN, Harris MB. Clinical practice. Lumbar spinal stenosis. N Engl J Med 2008;358:818-25.

4. Wu AM, Zou F, Cao Y, Xia DD, He W, Zhu B, Chen D, Ni WF, Wang XY, Kwan K. Lumbar spinal stenosis: an update on the epidemiology, diagnosis and treatment. AME Med J 2017;2:63.

5. Káplár Z, Wáng YJ. South Korean degenerative spondylolisthesis patients had surgical treatment at earlier age than Japanese, American, and European patients: a published literature observation. Quant Imaging Med Surg 2016;6:785-90. 
6. Wu AM, Hu ZC, Li XB, Feng ZH, Chen D, Xu H, Huang QS, Lin Y, Wang XY, Zhang K, Zhao J, Ni WF. Comparison of minimally invasive and open transforaminal lumbar interbody fusion in the treatment of single segmental lumbar spondylolisthesis: minimum two-year follow up. Ann Transl Med 2018;6:105.

7. Porter RW, Hibbert C, Evans C. The natural history of root entrapment syndrome. Spine 1984;9:418-21.

8. Kunogi J, Hasue M. Diagnosis and operative treatment of intraforaminal and extraforaminal nerve root compression. Spine 1991;16:1312-20.

9. Orita S, Inage K, Eguchi Y, Kubota G, Aoki Y, Nakamura J, Matsuura Y, Furuya T, Koda M, Ohtori S. Lumbar foraminal stenosis, the hidden stenosis including at L5/S1. Eur J Orthop Surg Traumatol 2016;26:685-93.

10. Hasegawa T, An HS, Haughton VM, Nowicki BH. Lumbar foraminal stenosis: critical heights of the intervertebral discs and foramina. A cryomicrotome study in cadavera. J Bone Joint Surg Am 1995;77:32-8.

11. Burton CV, Kirkaldy-Willis WH, Yong-Hing K, Heithoff $\mathrm{KB}$. Causes of failure of surgery on the lumbar spine. Clin Orthop Relat Res 1981;157:191-9.

12. Schofferman J, Reynolds J, Herzog R, Covington E, Dreyfuss P, O'Neill C. Failed back surgery: etiology and diagnostic evaluation. Spine J 2003;3:400-3.

13. Waguespack A, Schofferman J, Slosar P, Reynolds J. Etiology of long-term failures of lumbar spine surgery. Pain Med 2002;3:18-22.

14. Lee S, Lee JW, Yeom JS, Kim KJ, Kim HJ, Chung SK, Kang HS. A practical MRI grading system for lumbar foraminal stenosis. AJR Am J Roentgenol 2010;194:1095-8.

15. Williams AL, Gornet MF, Burkus JK. CT evaluation of lumbar interbody fusion: current concepts. AJNR Am J

Cite this article as: $\mathrm{Wu} \mathrm{AM}$, Zhang $\mathrm{K}, \mathrm{Li} \mathrm{XL}$, Cheng $\mathrm{XF}$, Zhou TJ, Du L, Chen C, Tian HJ, Sun XJ, Zhao CQ, Li YM, Zhao J. The compression of L5 nerve root, single or double sites?-radiographic graded signs, intra-operative detect technique and clinical outcomes. Quant Imaging Med Surg 2018;8(4):383-390. doi: 10.21037/qims.2018.05.08
Neuroradiol 2005;26:2057-66.

16. Fogel GR, Toohey JS, Neidre A, Brantigan JW. Fusion assessment of posterior lumbar interbody fusion using radiolucent cages: $\mathrm{X}$-ray films and helical computed tomography scans compared with surgical exploration of fusion. Spine J 2008;8:570-7.

17. Shah RR, Mohammed S, Saifuddin A, Taylor BA. Comparison of plain radiographs with CT scan to evaluate interbody fusion following the use of titanium interbody cages and transpedicular instrumentation. Eur Spine J 2003;12:378-85.

18. Singh V, Montgomery SR, Aghdasi B, Inoue H, Wang JC, Daubs MD. Factors affecting dynamic foraminal stenosis in the lumbar spine. Spine J 2013;13:1080-7.

19. Sakai $Y$, Ito S, Hida T, Ito K, Harada A, Watanabe K. Clinical outcome of lumbar spinal stenosis based on new classification according to hypertrophied ligamentum flavum. J Orthop Sci 2017;22:27-33.

20. Griffith JF, Huang J, Law SW, Xiao F, Leung JC, Wang D, Shi L. Population reference range for developmental lumbar spinal canal size. Quant Imaging Med Surg 2016;6:671-9.

21. Macnab I. Negative Disc Exploration: an analysis of the causes of nerve-root involvement in sixty-eight patients. J BONE JOINT SURG AM 1971;53:891-903.

22. Shiraishi T, Crock H. Re-exploration of the lumbar spine following simple discectomy: a review of 23 cases. Eur Spine J 1995;4:84-7.

23. Jeong TS, Ahn Y, Lee SG, Kim WK, Son S, Kwon JH. Correlation between MRI Grading System and Surgical Findings for Lumbar Foraminal Stenosis. J Korean Neurosurg Soc 2017;60:465-70. 